\title{
Abemaciclib plus fulvestrant in hormone receptor-positive, human epidermal growth factor receptor 2-negative advanced breast cancer in premenopausal women: subgroup analysis from the MONARCH 2 trial
}

Patrick Neven ${ }^{1 *}$, Hope S. Rugo ${ }^{2}$, Sara M. Tolaney ${ }^{3}$, Hiroji Iwata ${ }^{4}$, Masakazu Toi ${ }^{5}$, Matthew P. Goetz ${ }^{6}$, Peter A. Kaufman ${ }^{7}$, Yi Lu $^{8}$, Nadine Haddad ${ }^{8}$, Karla C. Hurt ${ }^{8}$ and George W. Sledge Jr. ${ }^{9}$

\begin{abstract}
Background: In MONARCH 2, abemaciclib plus fulvestrant significantly improved median progression-free survival (PFS, 16.4 vs 9.3 months, hazard ratio [HR] 0.553) and overall survival (OS, 46.7 vs 37.3 months; HR 0.757) compared with placebo plus fulvestrant in hormone receptor-positive (HR-positive), human epidermal growth factor receptor 2-negative (HER2-negative) advanced breast cancer (ABC) patients who were endocrine therapy (ET) resistant, regardless of menopausal status. Here, we report findings in the premenopausal subgroup of the MONARCH 2 trial.

Methods: The premenopausal subgroup included patients with natural menstrual bleeding who received a gonadotropin-releasing hormone agonist at least 4 weeks prior to study treatment start date and for the entire study duration. Of the 669 patients enrolled in the MONARCH 2 trial, 114 were premenopausal (abemaciclib plus fulvestrant, $n=72$; placebo plus fulvestrant, $n=42$ ), and were included in this analysis. The primary objective was investigatorassessed PFS and secondary objectives were OS, objective response rate, and safety and tolerability. Exploratory analyses included time to second disease progression (PFS2), time to chemotherapy (TTC), and chemotherapy-free survival (CFS).

Results: At the primary objective cutoff (February 14, 2017), median PFS was not reached for the abemaciclib plus fulvestrant arm versus 10.52 months for the placebo plus fulvestrant arm ( $H R 0.415 ; 95 \% \mathrm{Cl} 0.246-0.698)$. At the prespecified OS interim cutoff (20-June-2019), median PFS was 28.6 months in the abemaciclib plus fulvestrant arm compared with 10.26 months in the placebo plus fulvestrant arm ( $\mathrm{HR} 0.477 ; 95 \% \mathrm{Cl} 0.302-0.755)$. A numerical OS benefit was observed with abemaciclib plus fulvestrant compared to fulvestrant alone (HR 0.689; 95\% Cl 0.379-1.252, median, not reached vs 47.3 months). Improvements were also observed for the exploratory outcomes of PFS2 (HR 0.599), TTC (HR 0.674), and CFS (HR 0.642) with the addition of abemaciclib to fulvestrant. The safety profile was generally consistent with results disclosed previously.
\end{abstract}

*Correspondence: patrick.neven@uzleuven.be

${ }^{1}$ Department of Oncology, University Hospitals Leuven, KU Leuven-

University of Leuven, Herestraat 49, 3000 Leuven, Belgium

Full list of author information is available at the end of the article

(c) The Author(s) 2021. Open Access This article is licensed under a Creative Commons Attribution 4.0 International License, which permits use, sharing, adaptation, distribution and reproduction in any medium or format, as long as you give appropriate credit to the original author(s) and the source, provide a link to the Creative Commons licence, and indicate if changes were made. The images or other third party material in this article are included in the article's Creative Commons licence, unless indicated otherwise in a credit line to the material. If material is not included in the article's Creative Commons licence and your intended use is not permitted by statutory regulation or exceeds the permitted use, you will need to obtain permission directly from the copyright holder. To view a copy of this licence, visit http://creativecommons.org/licenses/by/4.0/. The Creative Commons Public Domain Dedication waiver (http://creativeco mmons.org/publicdomain/zero/1.0/) applies to the data made available in this article, unless otherwise stated in a credit line to the data. 
Conclusions: Results of the premenopausal subgroup in the MONARCH 2 trial were consistent with the improved clinical outcomes observed in the intent-to-treat population. The analysis provides support for the use of abemaciclib plus fulvestrant (with ovarian suppression) as an effective treatment option for premenopausal patients with HR+, HER2- ABC who are ET-resistant.

Clinical trial registration: NCT02107703. Registered April 08, 2014- Retrospectively registered, https:/clinicaltrials.gov/ ct2/show/NCT02107703.

Keywords: Abemaciclib, Advanced breast cancer, CDK4 and 6 inhibitor, Fulvestrant, Premenopausal women

\section{Background}

In recent years, the incidence of advanced breast cancer $(\mathrm{ABC})$ in women aged 25 to 39 years has slightly increased [1] and the disease is often more aggressive, with a poorer prognosis, and high rates of recurrence and mortality $[2,3]$. Hence, there is growing interest in appropriate treatment strategies for pre-/perimenopausal women (hereafter referred to as premenopausal women) with hormone receptor-positive $(\mathrm{HR}+)$, human epidermal growth factor receptor 2-negative (HER2-) ABC [2, 4-6]. This population subgroup is often under-represented in clinical trials $[3,6,7]$.

An optimal treatment approach in young women with $\mathrm{HR}+, \mathrm{HER} 2-\mathrm{ABC}$ is still poorly defined and treatment strategies are usually derived from data from postmenopausal patients. Studies have highlighted that given the differences in tumor biology and quality of life, extending treatment options across patients with different menopausal status might not be the best strategy $[2,7$, 8]. Additionally, there is the potential that these young women are at risk of over-treatment, based solely on age considerations [9].

Cyclin-dependent kinase 4 and 6 (CDK4 and 6) inhibitors have led to substantial improvements in progression-free survival (PFS) in the $A B C$ setting, resulting in approval of CDK4 and 6 inhibitors in combination with ET or as monotherapy in ABC patients [10-14]. More recently, some studies have also demonstrated overall survival (OS) benefit, further establishing the effectiveness of these agents in the treatment of $\mathrm{ABC}[6,15,16]$. Some of these $[4,5,15]$ reported improved PFS in this premenopausal subgroup, leading to the recommendation of fulvestrant in combination with CDK4 and 6 inhibitors and ovarian suppression as a treatment option for premenopausal patients with HR+, HER2- ABC [17].

Abemaciclib is an oral, selective, and potent small molecule CDK4 and 6 dual inhibitor for the treatment of HR+, HER2- ABC that is dosed on a continuous schedule. Abemaciclib is currently approved in three indications in HR+, HER2- ABC; in combination with aromatase inhibitor for postmenopausal women as initial endocrine-based therapy (MONARCH 3) [12, 14], in combination with fulvestrant for women with disease progression following ET (MONARCH 2) [13], and as a single agent for patients with disease progression following ET and prior chemotherapy in the metastatic setting (MONARCH 1) [12, 14].

In the intent-to-treat (ITT) population (669 patients) of the MONARCH 2 trial, abemaciclib plus fulvestrant significantly improved investigator-assessed PFS compared with placebo plus fulvestrant in women with $\mathrm{HR}+$, HER2- ABC who were ET-resistant (median PFS, 16.4 vs 9.3 months; hazard ratio [HR], 0.553; 95\% confidence interval $[\mathrm{CI}], 0.449-0.681 ; P<0.001)$ [13]. Abemaciclib treatment resulted in improved objective response rate (ORR; $48.1 \%$ vs $21.3 \%$ in the control arm). The OS was significantly improved in the abemaciclib plus fulvestrant arm (median OS, 46.7 vs 37.3 months; HR 0.757; 95\% CI $0.606-0.945 ; P=0.01$ ) [15]. The addition of abemaciclib to ET led to consistent treatment benefit across subgroups including menopausal status. Here, we report the efficacy and safety results for the premenopausal patient subgroup from the MONARCH 2 trial.

\section{Methods}

\section{Study design and patients}

MONARCH 2 was a randomized, double-blind, phase III, trial of abemaciclib or placebo with fulvestrant in $\mathrm{HR}+$, HER2- ABC women with any menopausal status (pre-, peri-, or post-menopausal) who had disease progression following ET. Patients were required to have disease that progressed while receiving prior ET (neoadjuvant or adjuvant ET, $\leq 12$ months after completion of adjuvant $\mathrm{ET}$, or while receiving first-line ET for metastatic disease). Patients with more than one ET or any prior chemotherapy for $A B C$ were excluded. Full trial details and eligibility criteria were published previously [13].

This article presents the subanalysis of the premenopausal population which comprised patients with natural menstrual bleeding who received a gonadotropin releasing-hormone (GnRH) agonist, such as goserelin, at least 4 weeks prior to study treatment start date and for the entire study duration.

The study was approved by the ethical and local institutional review boards for the sites participating in the clinical trial, and was conducted in accordance with the 
Good Clinical Practice guidelines and the Declaration of Helsinki. All patients provided written informed consent before enrollment. This study was overseen by a steering committee, and safety data were evaluated quarterly by an independent data monitoring committee.

\section{Treatment procedure}

Details of the MONARCH 2 trial have been published previously [13]. Briefly, patients received abemaciclib (150 mg) or placebo twice daily during each 28 -day cycle plus $500 \mathrm{mg}$ fulvestrant (per label). Treatment continued until progressive disease, death, or discontinuation for any other reason.

\section{Efficacy and safety assessments}

Safety analysis was performed on all patients who received at least one dose of study drug. Adverse events (AEs) were graded according to the National Cancer Institute Common Terminology Criteria for Adverse Events (CTCAE), version 4.0, and were evaluated at every patient visit from baseline until follow-up.

\section{Endpoints}

The primary endpoint was investigator-assessed PFS as defined by Response Evaluation Criteria in Solid Tumors version 1.1, and measured from the time of randomized assignment until progressive disease or death (whichever was earlier). The secondary endpoints included OS (time of randomized assignment until death), ORR (proportion of patients with complete response or partial response $[\mathrm{PR}])$, and safety and tolerability. Exploratory endpoints included: PFS2, defined as time from randomization to discontinuation of first subsequent post-discontinuation therapy or death (whichever was earlier), TTC, defined as time from randomization to initiation of first post-discontinuation chemotherapy (censoring patients who died prior to initiation of chemotherapy), and CFS, defined as time from randomization to initiation of first postdiscontinuation chemotherapy or death (whichever was earlier).

\section{Statistical analyses}

The data cutoff for the primary analysis of PFS occurred on February 14, 2017. ORR, change in tumor size, and safety evaluations were also reported from the primary analysis. The data cutoff for the preplanned interim analysis of the key secondary endpoint of OS occurred on 20 June, 2019. At this interim data cutoff, PFS was updated and the exploratory endpoints of PFS2, TTC, and CFS were also evaluated descriptively.

All efficacy analyses were performed on the subgroup of premenopausal patients within the ITT population, corresponding to all premenopausal patients randomized to study treatment. For time-to-event endpoints, the Kaplan-Meier method was used to estimate the survival curve for each treatment group, and the Cox proportional hazard model was used to estimate the HR and corresponding 95\% CI with an interaction term of menopausal status with treatment group. An exploratory mixed-model analysis was used to compare change in tumor size over time. Unless otherwise noted, all confidence intervals used a 95\% confidence level. Safety was assessed in all premenopausal patients who received at least one dose of any study treatment (i.e. the safety population). The statistical analyses were performed using SAS (version 9.2 or later; SAS Institute, Cary, NC).

\section{Results}

\section{Patients and treatment}

In the MONARCH 2 trial, 669 patients were randomly assigned to receive abemaciclib plus fulvestrant $(n=446)$ or placebo plus fulvestrant $(n=223)$. Of these, 114 patients $(17.0 \%)$ were premenopausal (premenopausal population) and received ovarian suppression with a GnRH agonist. This included 72 patients in the abemaciclib plus fulvestrant arm, of whom 71 received study treatment, and 42 in the placebo plus fulvestrant arm.

Demographic and baseline characteristics were wellbalanced between the two treatment arms (Table 1). At baseline, 60 patients $(52.6 \%)$ presented with visceral disease and 34 (29.8\%) with bone-only disease. A total of 43 patients (37.7\%) had primary ET resistance (defined as relapse while on the first 2 years of adjuvant ET or PD within the first 6 months of first-line ET) [18].

At the primary PFS cutoff, a total of $40(55.6 \%)$ patients in the abemaciclib plus fulvestrant arm and 11 (26.2\%) patients in the placebo plus fulvestrant arm remained on treatment. The median number of cycles received was 17 in the abemaciclib plus fulvestrant arm and 11 in the placebo plus fulvestrant arm. At the data cutoff for interim OS analysis, 21 premenopausal patients $(29.2 \%)$ in the abemaciclib plus fulvestrant arm versus four patients (9.5\%) in the placebo plus fulvestrant arm were still receiving the study treatment. The median length of follow-up was approximately 4 years in each treatment arm (48.69 and 47.70 months in the abemaciclib plus fulvestrant and placebo plus fulvestrant arms, respectively).

\section{Progression-free survival}

At the time of the primary analysis cutoff, 28 (38.9\%) versus 29 (69.0\%) PFS events were observed and mPFS was not reached versus 10.52 months in the abemaciclib plus fulvestrant and placebo plus fulvestrant arms, respectively. The addition of abemaciclib to fulvestrant resulted in a meaningful improvement in PFS (HR 0.415; 95\% CI 0.246-0.698), Fig. 1A. The PFS benefit was confirmed by 
Table 1 Patient and disease baseline characteristics for premenopausal patients in the MONARCH 2 trial

\begin{tabular}{|c|c|c|}
\hline & $\begin{array}{l}\text { Abemaciclib + fulvestrant } \\
N=72\end{array}$ & $\begin{array}{l}\text { Placebo + fulvestrant } \\
N=42\end{array}$ \\
\hline Median age (range) & $46(32-57)$ & $47(32-66)$ \\
\hline \multicolumn{3}{|l|}{ Race, $n(\%)$} \\
\hline Asian & $51(70.8)$ & $24(57.1)$ \\
\hline Caucasian & $14(19.4)$ & $16(38.1)$ \\
\hline Other & $7(9.7)$ & $2(4.8)$ \\
\hline \multicolumn{3}{|l|}{ Most recent $\mathrm{ET}, n(\%)^{\mathrm{a}}$} \\
\hline Neoadjuvant or adjuvant & $44(61.1)$ & $21(50.0)$ \\
\hline Metastatic & $26(36.1)$ & $20(47.6)$ \\
\hline \multicolumn{3}{|l|}{ Number of lines of ET, $n(\%)$} \\
\hline 1 & $60(83.3)$ & $30(71.4)$ \\
\hline 2 & $10(13.9)$ & $11(26.2)$ \\
\hline \multicolumn{3}{|l|}{ Prior Al, $n$ (\%) } \\
\hline Yes & $10(13.9)$ & $12(28.6)$ \\
\hline No & $62(86.1)$ & $30(71.4)$ \\
\hline \multicolumn{3}{|l|}{ Sensitivity to $\mathrm{ET}, n(\%)^{\mathrm{a}}$} \\
\hline Primary resistance ${ }^{b}$ & $28(38.9)$ & $15(35.7)$ \\
\hline Secondary resistance ${ }^{c}$ & $42(58.3)$ & $26(61.9)$ \\
\hline \multicolumn{3}{|c|}{ Progesterone-receptor status, $n$ (\%) } \\
\hline Positive & $54(75.0)$ & $38(90.5)$ \\
\hline Negative & $18(25.0)$ & $4(9.5)$ \\
\hline \multicolumn{3}{|l|}{ Metastatic site, $n(\%)$} \\
\hline Visceral & $43(59.7)$ & $17(40.5)$ \\
\hline Bone only & $19(26.4)$ & $15(35.7)$ \\
\hline Other & $10(13.9)$ & $10(23.8)$ \\
\hline \multicolumn{3}{|l|}{ Measurable disease, $n(\%)$} \\
\hline Yes & $51(70.8)$ & $28(66.7)$ \\
\hline No & $21(29.2)$ & $14(33.3)$ \\
\hline \multicolumn{3}{|l|}{ ECOG performance status } \\
\hline 0 & $54(75.0)$ & $36(85.7)$ \\
\hline 1 & $18(25.0)$ & $6(14.3)$ \\
\hline
\end{tabular}

$\mathrm{Al}$, aromatase inhibitor; ECOG, Eastern Cooperative Oncology Group; ET, endocrine therapy

${ }^{a}$ Two patients in the abemaciclib arm and one patient in the placebo arm received no prior $\mathrm{ET} ;{ }^{\mathrm{b}}$ Patients whose disease relapsed $\leq 2$ years while receiving (neo) adjuvant $\mathrm{ET}$ or progressed $\leq 6$ months of receiving $\mathrm{ET}$ for $\mathrm{ABC} ;{ }^{\mathrm{C}}$ Patients receiving prior $\mathrm{ET}$ who do not meet the definition of primary resistance were considered to have secondary resistance

blinded independent central review (HR 0.432; 95\% CI 0.236-0.793).

At the interim OS analysis cutoff, 20 June 2019, the PFS analysis was updated. In the abemaciclib plus fulvestrant arm, 42 (58.3\%) PFS events were observed versus 33 (78.6\%) in the placebo plus fulvestrant arm. The median PFS achieved in the abemaciclib plus fulvestrant arm was 28.6 months compared with 10.26 months in the control arm (HR 0.477; 95\% CI 0.302 to 0.755), Fig. 1B.

\section{Objective response rate and tumor shrinkage}

ORR was higher in the abemaciclib plus fulvestrant arm compared to the placebo plus fulvestrant arm (43.1\%;
95\% CI 31.6-54.5 vs 19.0\%; 95\% CI 7.2-30.9) (Table 2). This included two $(2.8 \%)$ complete responses in the abemaciclib plus fulvestrant arm compared with no complete response in the placebo plus fulvestrant arm. Patients with measurable disease achieved an ORR of $60.8 \%$ (95\% CI 47.4-74.2) in the abemaciclib plus fulvestrant arm and $28.6 \%$ (95\% CI 11.8-45.3) in the placebo plus fulvestrant arm $(P=0.006)$. An exploratory analysis of mean change in tumor size demonstrated that after 12 cycles, tumor size in the abemaciclib plus fulvestrant arm decreased by $64.6 \%$ compared to $42.5 \%$ in the placebo arm. 


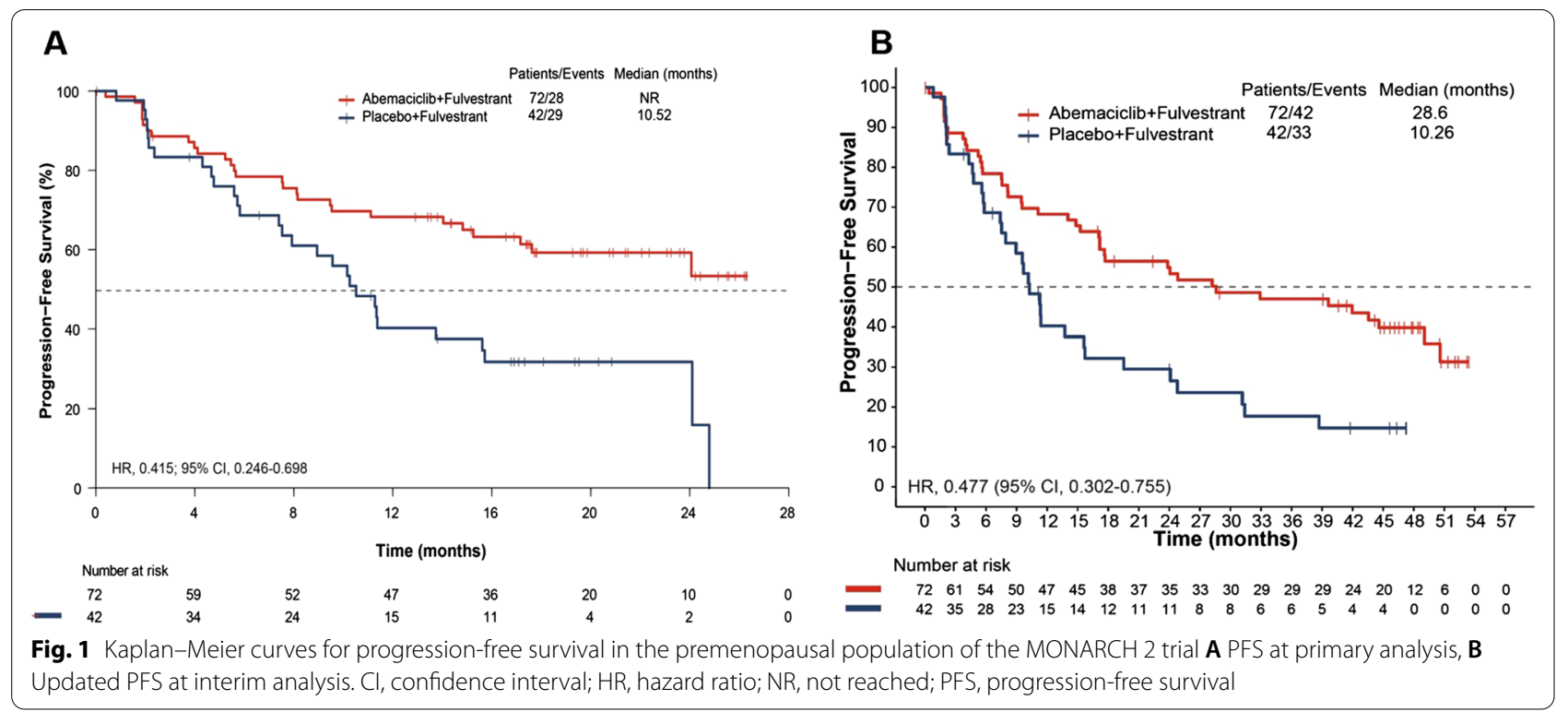

Table 2 Best overall response in the premenopausal population of the MONARCH 2 trial

\begin{tabular}{|c|c|c|c|c|c|}
\hline \multirow[t]{2}{*}{ Overall population } & \multicolumn{2}{|c|}{$\begin{array}{l}\text { Abemaciclib + fulvestrant } \\
N=72\end{array}$} & \multicolumn{2}{|c|}{$\begin{array}{l}\text { Placebo + fulvestrant } \\
N=42\end{array}$} & \multirow[t]{2}{*}{$P$ value } \\
\hline & $n(\%)$ & $95 \% \mathrm{Cl}$ & $n(\%)$ & $95 \% \mathrm{Cl}$ & \\
\hline$C R$ & $2(2.8)$ & $-1.0,6.6$ & $0(0)$ & - & \\
\hline$P R$ & $29(40.3)$ & $28.9,51.6$ & $8(19.0)$ & $7.2,30.9$ & \\
\hline SD & $30(41.7)$ & $30.3,53.1$ & $27(64.3)$ & $49.8,78.8$ & \\
\hline$\geq 6$ months & $25(34.7)$ & $23.7,45.7$ & $21(50.0)$ & $34.9,65.1$ & \\
\hline Progressive disease & $8(11.1)$ & $3.9,18.4$ & $6(14.3)$ & $3.7,24.9$ & \\
\hline Not evaluable & $3(4.2)$ & $-0.4,8.8$ & $1(2.4)$ & $-2.2,7.0$ & \\
\hline Overall response rate $(C R+P R)$ & $31(43.1)$ & $31.6,54.5$ & $8(19.0)$ & $7.2,30.9$ & .009 \\
\hline Disease control rate $(C R+P R+S D)$ & $61(84.7)$ & $76.4,93.0$ & $35(83.3)$ & $72.1,94.6$ & .845 \\
\hline Clinical benefit rate (CR+PR $+S D \geq 6$ months) & $56(77.8)$ & $68.2,87.4$ & $29(69.0)$ & $55.1,83.0$ & .304 \\
\hline \multirow[t]{2}{*}{ Measurable disease population } & \multicolumn{2}{|c|}{$\begin{array}{l}\text { Abemaciclib + fulvestrant } \\
N=51\end{array}$} & \multicolumn{2}{|c|}{$\begin{array}{l}\text { Placebo + fulvestrant } \\
N=28\end{array}$} & $P$ value \\
\hline & $n(\%)$ & $95 \% \mathrm{Cl}$ & $n(\%)$ & $95 \% \mathrm{Cl}$ & \\
\hline$C R$ & $2(3.9)$ & $-1.4,9.2$ & $0(0.0)$ & - & \\
\hline PR & $29(56.9)$ & $43.3,70.5$ & $8(28.6)$ & $11.8,45.3$ & \\
\hline SD & $11(21.6)$ & $10.3,32.9$ & $16(57.1)$ & $38.8,75.5$ & \\
\hline$\geq 6$ months & $7(13.7)$ & $4.3,23.2$ & $12(42.9)$ & $24.5,61.2$ & \\
\hline Progressive disease & $7(13.7)$ & $4.3,23.2$ & $4(14.3)$ & $1.3,27.2$ & \\
\hline Not evaluable & $2(3.9)$ & $-1.4,9.2$ & $0(0.0)$ & - & \\
\hline Overall response rate $(C R+P R)$ & $31(60.8)$ & $47.4,74.2$ & $8(28.6)$ & $11.8,45.3$ & .006 \\
\hline Disease control rate $(C R+P R+S D)$ & $42(82.4)$ & $71.9,92.8$ & $24(85.7)$ & $72.8,98.7$ & .702 \\
\hline Clinical benefit rate (CR+PR $+S D \geq 6$ months) & $38(74.5)$ & $62.5,86.5$ & $20(71.4)$ & $54.7,88.2$ & .768 \\
\hline
\end{tabular}

$\mathrm{Cl}$, confidence interval; $\mathrm{CR}$, complete response; ITT, intent-to-treat; $\mathrm{N}$, number of patients in the arm; $n$, number of patients in each subgroup; $\mathrm{PR}$, partial response; $\mathrm{SD}$, stable disease

* Using RECIST version 1.1 


\section{Overall survival}

At the interim OS cutoff, there were 25 (34.7\%) versus 19 (45.2\%) deaths and mOS was not reached versus 47.31 months in the abemaciclib plus fulvestrant versus placebo plus fulvestrant arms, respectively. A numerical OS benefit was observed with the addition of abemaciclib to fulvestrant (HR 0.689; 95\% CI 0.379-1.252; Fig. 2) which was consistent with the ITT population.

\section{Post-discontinuation therapy}

At the interim OS analysis, a total of 88 patients had discontinued study treatment including 50 (69.4\%) patients in the abemaciclib plus fulvestrant arm and 38 (90.5\%) patients in the placebo plus fulvestrant arm. Forty-one (56.9\%) patients in the abemaciclib plus fulvestrant arm compared to $35(83.3 \%)$ patients in the placebo plus fulvestrant arm received a post-discontinuation therapy. Chemotherapy was received by 60 patients $(n=34,47.2 \%$ patients vs $n=26,61.9 \%$ patients in the abemaciclib plus fulvestrant and placebo plus fulvestrant arms, respectively) at any time post-discontinuation. ET was received by 48 patients $(n=26,36.1 \%$ patients in the abemaciclib plus fulvestrant arm vs $n=22,52.4 \%$ patients in the placebo plus fulvestrant arm), while 41 patients received a targeted therapy $(n=19,26.4 \%$ patients in the abemaciclib plus fulvestrant arm vs $n=22,52.4 \%$ patients in the placebo plus fulvestrant arm), and 17 patients received other therapies $(n=9,12.5 \%$ patients in the abemaciclib plus fulvestrant arm vs $n=8,19.0 \%$ patients in the placebo plus fulvestrant arm). Among those who received targeted therapies, 15 patients received CDK 4 and 6 inhibitor(s) as post-discontinuation therapy $(n=5,6.9 \%$

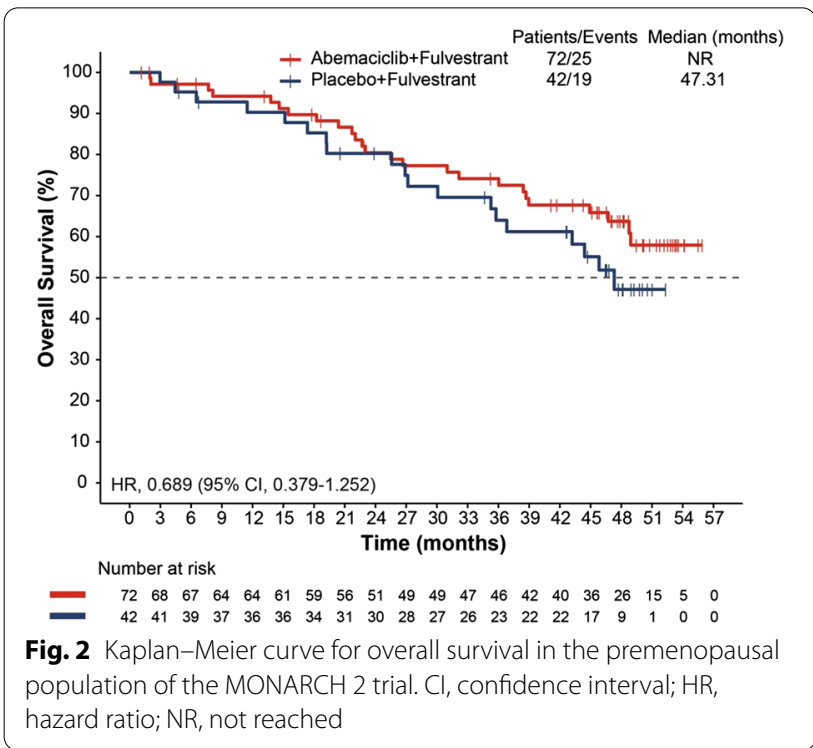

patients in the abemaciclib plus fulvestrant arm vs $n=10$, $23.8 \%$ patients in the placebo plus fulvestrant arm).

\section{Other exploratory endpoints}

With the addition of abemaciclib to fulvestrant, a clinically meaningful benefit was observed in other exploratory endpoints including PFS2 (HR 0.599; 95\% CI 0.371-0.968; Fig. 3A), TTC (HR 0.674; 95\% CI 0.4041.124; Fig. 3B) and CFS (HR 0.642; 95\% CI 0.398-1.037; Fig. 3C), consistent with ITT population.

Median PFS2 was 35.64 months in the abemaciclib plus fulvestrant arm versus 21.27 months in the placebo plus fulvestrant arm.

The median time to first chemotherapy treatment (censoring patients who died prior to receiving chemotherapy) was 50.24 months in the abemaciclib plus fulvestrant arm and 26.76 months in the placebo plus fulvestrant arm.

Deaths prior to receiving any chemotherapy were four (5.6\%) patients in the abemaciclib arm and 4 (9.5\%) patients in the placebo arm. Median CFS (including both chemotherapy and death as events) was 38.07 versus 19.07 months in the abemaciclib versus placebo arms, respectively.

\section{Safety}

The safety profile in the premenopausal subgroup was consistent to the ITT population. The most frequent adverse events of any grade were diarrhea $(87.3 \%$ vs $23.8 \%)$, neutropenia $(59.2 \%$ vs $7.1 \%)$ and leukopenia (43.7\% vs $4.8 \%)$ in the abemaciclib plus fulvestrant versus placebo plus fulvestrant arms, respectively (Table 3 ). In the abemaciclib arm, key grade 3 adverse events of special interest were neutropenia (39.4\%), leukopenia (16.9\%), and diarrhea (11.3\%). Serious adverse events were reported in eight patients $(11.3 \%)$ in the abemaciclib plus fulvestrant arm and two patients (4.8\%) in the placebo plus fulvestrant arm (Additional file 1: Supplemental Table 1).

Four patients (5.6\%) discontinued study treatment due to an $\mathrm{AE}$ (neutropenia, diarrhea, drug-induced liver injury, and increased aspartate aminotransferase, $n=1$ each). Twenty-eight (39.4\%) patients in the abemaciclib versus $1(2.4 \%)$ patient in the placebo arm had at least one dose reduction in abemaciclib/placebo due to an $\mathrm{AE}$. In the abemaciclib arm, the AEs leading to dose reduction included neutropenia $(n=14,19.7 \%)$, diarrhea $(n=12,16.9 \%)$, nausea $(n=2,2.8 \%)$, and lymphopenia $n=1,1.4 \%)$.

No deaths due to AEs, during or within 30 days after treatment, occurred in the premenopausal population. At the primary PFS lock, one pre-menopausal subject was reported as death (within 30 days of treatment 


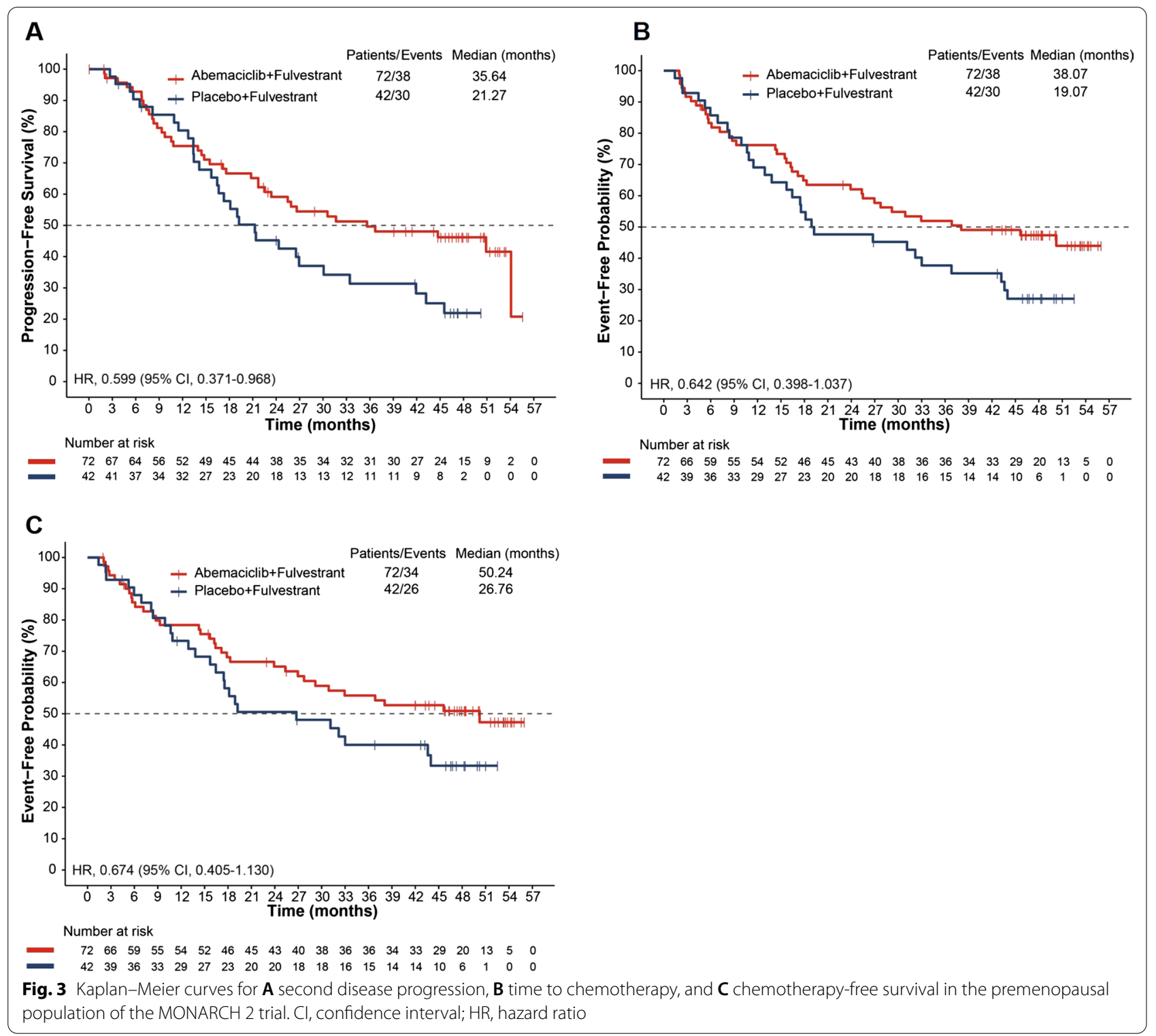

discontinuation) due to grade 5 embolism event reported as cerebral infarction. Later, at the OS interim lock, the investigator re-evaluated the primary death reason to be study disease.

\section{Discussion}

MONARCH 2 study reported a large absolute OS benefit in a phase III clinical trial for HR+, HER2- ABC, regardless of menopausal status $[6,16]$. In this report, we evaluated the treatment benefit of abemaciclib plus fulvestrant in the premenopausal subgroup of the MONARCH 2 trial. Our findings indicate that premenopausal patients with ET-resistant, HR+, HER2- ABC derived benefit from the addition of abemaciclib to fulvestrant, with outcomes broadly consistent with those in the overall ITT population.

In this subset study, PFS was prolonged in premenopausal subgroup (HR 0.415) with the addition of abemaciclib to fulvestrant. Additionally, the observed numerical OS benefit in this subgroup (HR 0.689) was consistent with findings from the ITT population (HR 0.757).

Improvements in favor of the abemaciclib arm were observed for all exploratory endpoints including PFS2, TTC and CFS, also consistent with the ITT population [15], further strengthening our understanding of the treatment benefit with abemaciclib. The improvement in PFS2 demonstrated the treatment effect of abemaciclib 
Table 3 Treatment-emergent adverse events occurring in $\geq 10 \%$ of the premenopausal population of the MONARCH 2 trial

\begin{tabular}{|c|c|c|c|c|c|c|c|c|}
\hline \multirow[t]{2}{*}{ Adverse events ( $\geq 10 \%$ patients in either arm) } & \multicolumn{4}{|c|}{$\begin{array}{l}\text { Abemaciclib + fulvestrant } \\
N=71\end{array}$} & \multicolumn{4}{|c|}{$\begin{array}{l}\text { Placebo + fulvestrant } \\
N=42\end{array}$} \\
\hline & All & G2 & G3 & G4 & All & G2 & G3 & G4 \\
\hline Any, $n(\%)$ & $70(98.6)$ & $20(28.2)$ & $40(56.3)$ & $4(5.6)$ & $40(95.2)$ & $17(40.5)$ & $7(16.7)$ & 0 \\
\hline Diarrhea & $62(87.3)$ & $22(31.0)$ & $8(11.3)$ & 0 & $10(23.8)$ & $1(2.4)$ & 0 & 0 \\
\hline Neutropenia ${ }^{a}$ & $42(59.2)$ & $9(12.7)$ & $28(39.4)$ & $2(2.8)$ & $3(7.1)$ & $1(2.4)$ & $1(2.4)$ & 0 \\
\hline Leukopenia & $31(43.7)$ & $15(21.1)$ & $12(16.9)$ & 0 & $2(4.8)$ & $1(2.4)$ & 0 & 0 \\
\hline Infections and infestations & $31(43.7)$ & $26(36.6)$ & $1(1.4)$ & 0 & $11(26.2)$ & $7(16.7)$ & $2(4.8)$ & 0 \\
\hline Abdominal pain & $25(35.2)$ & $2(2.8)$ & 0 & 0 & $5(11.9)$ & $2(4.8)$ & 0 & 0 \\
\hline Anemia & $24(33.8)$ & $15(21.1)$ & $7(9.9)$ & 0 & $1(2.4)$ & 0 & 0 & 0 \\
\hline Headache & $24(33.8)$ & $7(9.9)$ & - & 0 & $13(31.0)$ & $2(4.8)$ & 0 & 0 \\
\hline Vomiting & $23(32.4)$ & $5(7.0)$ & $1(1.4)$ & 0 & $3(7.1)$ & 0 & $1(2.4)$ & 0 \\
\hline Nausea & $20(28.2)$ & $6(8.5)$ & $1(1.4)$ & 0 & $10(23.8)$ & $3(7.1)$ & $1(2.4)$ & 0 \\
\hline Respiratory disorders & $16(22.5)$ & $4(5.6)$ & $1(1.4)$ & 0 & $9(21.4)$ & $1(2.4)$ & 0 & 0 \\
\hline Pyrexia & $13(18.3)$ & 0 & 0 & 0 & $6(14.3)$ & $1(2.4)$ & 0 & 0 \\
\hline Stomatitis & $13(18.3)$ & $3(4.2)$ & 0 & 0 & $9(21.4)$ & $2(4.8)$ & 0 & 0 \\
\hline Pruritus & $13(18.3)$ & $1(1.4)$ & 0 & 0 & $2(4.8)$ & 0 & 0 & 0 \\
\hline Thrombocytopenia & $12(16.9)$ & $4(5.6)$ & 0 & $2(2.8)$ & - & - & - & - \\
\hline Alanine aminotransferase increased & $11(15.5)$ & $4(5.6)$ & $3(4.2)$ & 0 & $2(4.8)$ & 0 & 0 & 0 \\
\hline Rash & $11(15.5)$ & $2(2.8)$ & $1(1.4)$ & 0 & - & - & - & - \\
\hline Constipation & $10(14.1)$ & $1(1.4)$ & $1(1.4)$ & 0 & $7(16.7)$ & 0 & 0 & 0 \\
\hline Arthralgia & $10(14.1)$ & $2(2.8)$ & $1(1.4)$ & 0 & $7(16.7)$ & $2(4.8)$ & 0 & 0 \\
\hline Injection site reaction & $10(14.1)$ & $1(1.4)$ & 0 & 0 & $5(11.9)$ & 0 & 0 & 0 \\
\hline Dry skin & $10(14.1)$ & $1(1.4)$ & 0 & 0 & - & - & - & - \\
\hline Fatigue & $9(12.7)$ & $3(4.2)$ & 0 & 0 & $10(23.8)$ & $4(9.5)$ & 0 & 0 \\
\hline Edema peripheral & $9(12.7)$ & $1(1.4)$ & 0 & 0 & $2(4.8)$ & 0 & 0 & 0 \\
\hline Hot flush & $8(11.3)$ & $2(2.8)$ & 0 & 0 & $3(7.1)$ & $2(4.8)$ & 0 & 0 \\
\hline Alopecia & $8(11.3)$ & $1(1.4)$ & 0 & 0 & - & - & - & - \\
\hline Aspartate aminotransferase increased & $8(11.3)$ & $4(5.6)$ & $1(1.4)$ & 0 & $4(9.5)$ & $1(2.4)$ & 0 & 0 \\
\hline
\end{tabular}

G, grade

a 1 patient (1.4\%) experienced grade 3 febrile neutropenia in the abemaciclib arm

carried over beyond the first disease progression to the subsequent line of therapy, which is of clinical relevance. Treatment with abemaciclib delayed chemotherapy and the median TTC was almost doubled in the abemaciclib plus fulvestrant arm compared to placebo plus fulvestrant arm. Post-discontinuation therapy was well-balanced considering the number of patients remaining on study treatment in the abemaciclib plus fulvestrant arm. The higher percentage of patients receiving a post-discontinuation therapy in the placebo arm is linked to the fact that a higher percentage of patients on the placebo arm discontinued study treatment. Of note, although the difference in median TTC is substantial, the absolute median in each arm for TTC should not be over-interpreted due to a lack of adjustment for patient death, as patients who died prior to receiving chemotherapy were censored.
The safety profile of the premenopausal subgroup was similar to that of the ITT population. Overall, treatment was well-tolerated, and diarrhea associated with abemaciclib was generally predictable (occurred early), manageable (with conventional doses of antidiarrheal medication and dose reduction), and reversible. No differences in the safety profile were observed in premenopausal women compared to postmenopausal women in the overall population.

The effect of CDK4 and 6 inhibitors on premenopausal patients with $A B C$ has been previously studied in two other trials. The combination of ribociclib plus ET (nonsteroidal aromatase inhibitor, ortamoxifen), evaluated in the MONALEESA-7 trial in a premenopausal population, demonstrated an improved PFS (median, 23.8 vs 13.0 months; HR 0.55; $P<0.0001$ ) [4] and OS (HR 0.71; 95\% CI 0.54-0.95; 4-year OS follow-up, HR 0.76; 95\% CI 0.61-0.96) [6, 19]. In contrast, the PALOMA 3 trial 
evaluating another CDK4 and 6 inhibitor, palbociclib, in combination with fulvestrant did not demonstrate an OS benefit among premenopausal patients (HR 1.07; 95\% CI 0.61 to $1.86 ; P=0.25$ ) [16]. This finding was consistent with the results in the ITT population, where no OS benefit was observed.

Notably, patient population, inclusion criteria and prior treatments differed among the MONARCH 2, MONALEESA-7, and PALOMA-3 trials. The MONALEESA-7 trial only included premenopausal women with $\leq 1$ line of prior chemotherapy for $A B C$ and $40 \%$ had de novo metastases [4]. The PALOMA-3 trial included both postmenopausal and premenopausal women, and $75 \%$ overall had prior ET or chemotherapy for $\mathrm{ABC}$ (prior ET, 46.1\%; prior chemotherapy, 34.0\%) [5]. In the MONARCH 2 trial, postmenopausal and premenopausal women with no prior chemotherapy for $A B C$ were enrolled, and $38 \%$ had 1 line of $E T$ for $A B C$. These differences should be considered while interpreting and comparing results among these studies.

A potential limitation of this analysis is the limited sample size. Given that formal statistical testing was not planned within the premenopausal subgroup, the focus of this analysis was to estimate the key efficacy parameters and to describe the safety profile in this patient population. Therefore, these results within premenopausal patients need to be interpreted with caveats for the MONARCH 2 trial as well as in the context of any cross-trial comparisons. Nonetheless, we observed that the magnitude of the treatment benefit in the premenopausal subgroup is comparable to that in the ITT population, suggesting a clinically meaningful improvement. Follow-up is ongoing to further characterize the longterm benefit.

\section{Conclusion}

Current clinical guidelines recommend that patients with HR+, HER2- disease should be treated preferentially with ET, and chemotherapy reserved for rapidly progressing, or symptomatic disease. There is an unmet need for defined treatment guidelines in premenopausal breast cancer patients. Consistent with the ITT population, the benefit of abemaciclib plus fulvestrant was maintained across premenopausal women with HR+, HER2- ABC in the MONARCH 2 trial. The data support extending the use of abemaciclib plus endocrine therapy for the treatment of premenopausal women with endocrine-resistant disease.

\section{Abbreviations}

ABC: Advanced breast cancer; CDK4 and 6: Cyclin-dependent kinase 4 and 6; CFS: Chemotherapy-free survival; Cl: Confidence interval; ET: Endocrine therapy; GnRH: Gonadotropin releasing-hormone; HER2-: Human epidermal growth factor receptor 2-negative; HR: Hazard ratio; HR+: Hormone receptorpositive, ITT, intent-to-treat; ITT: Intent-to-treat; ORR: Objective response rate; OS: Overall survival; PFS: Progression-free survival; PFS2: Second disease progression; TTC: Time to chemotherapy.

\section{Supplementary Information}

The online version contains supplementary material available at https://doi. org/10.1186/s13058-021-01463-2.

Additional file 1: Supplemental Table 1. Serious adverse events in the premenopausal population of the MONARCH 2 trial.

\section{Acknowledgements}

We thank the patients, caregivers, and investigators who participated in the MONARCH 2 trial. All authors meet the International Committee of Medical Journal Editors (ICMJE) criteria for authorship for this article, take responsibility for the integrity of the work as a whole, and have given their approval for this manuscript to be published. Medical Writing support and editorial support, funded by Eli Lilly and Company, was provided by Anchal Sood and Antonia Baldo, respectively, from Syneos Health.

\section{Authors' contributions}

$\mathrm{YL}, \mathrm{NH}$, and $\mathrm{KCH}$ contributed to the analysis and interpretation of data. All authors read and approved the final manuscript.

\section{Funding}

This work was funded by Eli Lilly and Company. The study sponsor provided the study drug and collaborated with the investigators to design the study; collect, analyze, and interpret the data; and write this report. The corresponding author had access to study data, and all authors approved submission for publication.

\section{Availability of data and materials}

Eli Lilly and Company provides access to all individual participant data collected during the trial, after anonymization, except for pharmacokinetic or genetic data. Data are available to request 6 months after the indication studied has been approved in the USA and EU and after primary publication acceptance, whichever is later. No expiration date of data requests is currently set once data are made available. Access is provided after a proposal has been approved by an independent review committee identified for this purpose and after receipt of a signed data sharing agreement. Data and documents, including the study protocol, statistical analysis plan, clinical study report, and blank or annotated case report forms, will be provided in a secure data sharing environment. For details on submitting a request, see the instructions provided at http://www.vivli.org.

\section{Declarations}

\section{Ethics approval and consent to participate}

The study was conducted in compliance with the ethical principles originating in or derived from the Declaration of Helsinki and in compliance with the International Council for Harmonization Good Clinical Practice Guidelines. All patients provided written informed consent. The final protocol, any amendments, and informed consent documentations were reviewed and approved by the Institutional Review Boards at each of the investigational centers participating in the study.

\section{Consent for publication}

Not applicable.

\section{Competing interests}

PN declares no conflicts of interest. HSR reports research grant to her institution from Eli Lilly and Company, Pfizer, Merck, Novartis, Genentech, OBI, Odonate, Daiichi-Sankyo, Eisai, Seattle Genetics, Macrogenics, Sermonixs, Immunomedics, AstraZeneca; travel support from Daiichi- Sankyo, Mylan, Pfizer, Merck, Novartis, AstraZeneca, Macrogenics; and honoraria from Puma, 
Mylan, and Samsung. SMT reports research grants to her institute and personal fees (honoraria for consulting/advisory board) from Eli Lilly and Company, AstraZeneca, Merck, Nektar, Novartis, Pfizer, Genentech/Roche, Exelixis, Bristol-Myers Squibb, Eisai, Nanostring, Sanofi, Odonate, Immunomedics/ Gilead; honoraria for advisory board/consulting from Puma, Celldex, Seattle Genetics, Silverback Therapeutics, G1 Therapeutics, Abbvie, Athenex, OncoPep, Kyowa Kirin Pharmaceuticals, Daiichi-Sankyo, CytomX, Samsung Bioepsis Inc., Certara, Mersana Therapeutics, OncoSec; and research funding from Cyclacel. HI reports honoraria and consulting fees from Chugai, Novartis, AstraZeneca, Pfizer, Eli Lilly and Company, Daichi-Sankyo, Eisai, Kyowa Hakko Kirin; grants from MSD, Bayer, Nihon Kayaku, Boehringer Ingelheim, Sanofi; and personal fees from Taiho. MT reports research grant and honoraria from Chugai, Takeda, Pfizer, Kyowa-Kirin, Taiho, Eisai, AstraZeneca, Daiichi-Sankyo, Shimadzu, Yakult, and Nippon Kayaku; research grant from JBCRG association, Astellas, AFI technologies, Shionogi, and GL Science; advisory role at Kyowa-Kirin, Daiichi-Sankyo, Eli Lilly and Company, Konica Minolta, Athenex Oncology, Bertis, Terumo, Luxonus, and Kansai Medical Net; honoraria from BMS, Eli Lilly and Company, MSD, Exact Science, Novartis, and Konica Minolta. He is also on the Board of Directors for DBCRG association, Organization for Oncology and Translational Research, Kyoto Breast Cancer Research Network. MPG reports consulting fees to his institution from Eagle pharmaceuticals, Biovica, Novartis, Context Therapeutics, Biotheranostics, and AstraZeneca; research grants and consulting fees to his institution from Eli Lilly and Company, Pfizer, and Sermonix. PAK reports grants from Eli Lilly and Company. YL, NH, and KCH are employees of Eli Lilly and Company. GWS reports grants and nonfinancial support from Stanford University during the conduct of the study; personal fees from Radius Pharmaceuticals and Verseau Therapeutics, grants from Pfizer, personal fees from Symphogen, personal fees and nonfinancial support from Tessa, and personal fees from Syndax outside the submitted work.

\section{Author details}

${ }^{1}$ Department of Oncology, University Hospitals Leuven, KU Leuven-University of Leuven, Herestraat 49, 3000 Leuven, Belgium. ${ }^{2}$ Department of Medicine (Hematology/Oncology), University of California San Francisco Helen Diller Family Comprehensive Cancer Center, San Francisco, CA, USA. ${ }^{3}$ Department of Medical Oncology, Dana-Farber Cancer Institute, Boston, MA, USA. ${ }^{4}$ Department of Breast Oncology, Aichi Cancer Center Hospital, Nagoya, Japan. ${ }^{5}$ Department of Breast Surgery, Graduate School of Medicine, Kyoto University, Kyoto, Japan. ${ }^{6}$ Department of Oncology, Mayo Clinic, Rochester, MN, USA. ${ }^{7}$ Department of Hematology and Oncology, Norris Cotton Cancer Center at Dartmouth-Hitchcock Medical Center, Lebanon, NH, USA. ${ }^{8}$ Eli Lilly and Company, Indianapolis, IN, USA. ${ }^{9}$ Department of Medicine, Stanford University School of Medicine, Stanford, CA, USA.

Received: 8 June 2021 Accepted: 4 August 2021

Published online: 23 August 2021

\section{References}

1. Johnson $\mathrm{RH}$, Chien FL, Bleyer A. Incidence of breast cancer with distant involvement among women in the United States, 1976 to 2009. JAMA. 2013;309:800-5.

2. Bardia A, Hurvitz S. Targeted therapy for premenopausal women with $\mathrm{HR}(+)$, HER2(-) advanced breast cancer: focus on special considerations and latest advances. Clin Cancer Res. 2018;24:5206-18.

3. Dalal AA, Gauthier G, Gagnon-Sanschagrin P, Burne R, Guérin A, Niravath $P$, et al. Treatment and monitoring patterns among premenopausal women with HR+/HER2- advanced breast cancer. Adv Ther. 2018;35:1356-67.

4. Tripathy D, Im SA, Colleoni M, Franke F, Bardia A, Harbeck N, et al. Ribociclib plus endocrine therapy for premenopausal women with hormonereceptor-positive, advanced breast cancer (MONALEESA-7): a randomised phase 3 trial. Lancet Oncol. 2018;19:904-15.
5. Loibl S, Turner NC, Ro J, Cristofanilli M, Iwata H, Im SA, et al. Palbociclib combined with fulvestrant in premenopausal women with advanced breast cancer and prior progression on endocrine therapy: PALOMA-3 results. Oncologist. 2017;22:1028-38.

6. Im SA, Lu YS, Bardia A, Harbeck N, Colleoni M, Franke F, et al. Overall survival with ribociclib plus endocrine therapy in breast cancer. N Engl J Med. 2019;381:307-16.

7. Tancredi R, Furlanetto J, Loibl S. Endocrine therapy in premenopausal hormone receptor positive/human epidermal growth receptor 2 negative metastatic breast cancer: between guidelines and literature. Oncologist. 2018;23:974-81.

8. Azim HA Jr, Partridge AH. Biology of breast cancer in young women. Breast Cancer Res. 2014;16:427.

9. Paluch-Shimon S, Pagani O, Partridge AH, Abulkhair O, Cardoso MJ, Dent RA, et al. ESO-ESMO 3rd international consensus guidelines for breast cancer in young women (BCY3). Breast. 2017;35:203-17.

10. Cristofanilli M, Turner NC, Bondarenko I, Ro J, Im SA, Masuda N, et al. Fulvestrant plus palbociclib versus fulvestrant plus placebo for treatment of hormone-receptor-positive, HER2-negative metastatic breast cancer that progressed on previous endocrine therapy (PALOMA-3): final analysis of the multicentre, double-blind, phase 3 randomised controlled trial. Lancet Oncol. 2016;17:425-39.

11. Hortobagyi GN, Stemmer SM, Burris HA, Yap YS, Sonke GS, Paluch-Shimon $\mathrm{S}$, et al. Updated results from MONALEESA-2, a phase III trial of first-line ribociclib plus letrozole versus placebo plus letrozole in hormone receptor-positive, HER2-negative advanced breast cancer. Ann Oncol. 2018;29:1541-7.

12. Dickler MN, Tolaney SM, Rugo HS, Cortés J, Diéras V, Patt D, et al. MONARCH 1, A phase II study of abemaciclib, a CDK4 and CDK6 inhibitor, as a single agent, in patients with refractory $\mathrm{HR}(+) / \mathrm{HER} 2(-)$ metastatic breast cancer. Clin Cancer Res. 2017;23:5218-24.

13. Sledge GW Jr, Toi M, Neven P, Sohn J, Inoue K, Pivot X, et al. MONARCH 2: abemaciclib in combination with fulvestrant in women with HR+/HER2advanced breast cancer who had progressed while receiving endocrine therapy. J Clin Oncol. 2017;35:2875-84.

14. Goetz MP, Toi M, Campone M, Sohn J, Paluch-Shimon S, Huober J, et al. MONARCH 3: abemaciclib as initial therapy for advanced breast cancer. J Clin Oncol. 2017:35:3638-46.

15. Sledge GW Jr, Toi M, Neven P, Sohn J, Inoue K, Pivot X, et al. The effect of abemaciclib plus fulvestrant on overall survival in hormone receptor-positive, ERBB2-negative breast cancer that progressed on endocrine therapy-MONARCH 2: a randomized clinical trial. JAMA Oncol. 2019;6:116-24

16. Turner NC, Slamon DJ, Ro J, Bondarenko I, Im SA, Masuda N, et al. Overall survival with palbociclib and fulvestrant in advanced breast cancer. $\mathrm{N}$ Engl J Med. 2018;379:1926-36.

17. National Comprehensive Cancer Network. NCCN Clinical Practice Guidelines in Oncology: Breast Cancer Version 6.2020. https://www.nccn.org/ professionals/physician_gls/pdf/breast.pdf. Accessed 20 Dec 2020.

18. Cardoso F, Paluch-Shimon S, Senkus E, Curigliano G, Aspro MS, Andre F, et al. 5th ESO-ESMO international consensus guidelines for advanced breast cancer (ABC 5). Ann Oncol. 2020;31(12):1623-49.

19. Tripathy D, Im SA, Colleoni M, Franke F, Bardia A, Harbeck N et al. Updated overall survival (OS) results from the phase III MONALEESA-7 trial of preor perimenopausal patients with hormone receptor positive/human epidermal growth factor receptor 2 negative (HR+/HER2-) advanced breast cancer $(A B C)$ treated with endocrine therapy $(E T) \pm$ ribociclib. 2020 San Antonio Breast Cancer Symposium, 2020. https://www.sabcs. org/Portals/SABCS2016/2020\%20SABCS/ALL\%20ABSTRACTS\%202-9.pdf? ver $=2020-12-09-104626-337$

\section{Publisher's Note}

Springer Nature remains neutral with regard to jurisdictional claims in published maps and institutional affiliations. 